\title{
Spectral characteristics during dip in Cyg X-1
}

\author{
Keisuke Sudoh ${ }^{1}$, Takayoshi Kohmura ${ }^{2}$ and Shunji Kitamoto ${ }^{1}$ \\ ${ }^{1}$ Department of Physics, Rikkyo University, 3-34-1 Nishi-Ikebukuro, Toshima-ku, Tokyo \\ 171-8501, Japan \\ 2 Physics Department, Kogakuin University, 2665-1 Nakano-cho, Hachioji, Tokyo, 192-0015, \\ Japan \\ E-mail: sudoh@stu.rikkyo.ne.jp, tkohmura@map.kogakuin.ac.jp, \\ kitamoto@ikkyo.ac.jp
}

We report the results of spectral analysis for black hole binary Cyg X-1, especially focussed on intensity dips. We used data otained with RXTE from May 5, 2000 for one orbital cycle, and in that time, many intensity dips are found. The dip spectrum are reduced by $\sim 2 \%$ from non-dip spectrum even in the 20-150 keV. We fitted dip and non-dip spectra with two CompPS model, which expresses thermal comptonizaiton of emission from accretion disk by hot electron cloud with defferent optical depth. During dip, column density for softer component is significantly larger than that for harder one, which suggests intensity dip is caused by absorption of softer component.

VII Microquasar Workshop: Microquasars and Beyond September 1 - 5, 2008

Foca, Izmir, Turkey 


\section{Introduction}

Cyg X-1 is the most famous Galactic black hole, accompanying a OIab type star with an orbital period of 5.6 days (Brocksopp et al. 1999). In Cyg X-1, intensity dips, the decrease of X-ray intensity, are often observed (Kitamoto et al. 1984; Feng \& Cui 2002). The dips occurred frequently near superior conjunction (phase 0), indicating that their cause is an absorption of the $\mathrm{X}$-rays from the nearby region of the black hole by a medium around companion star.

Cyg X-1 stays most of time in the low/hard state, its X-ray spectrum is basically described by a single power law, with some features: a soft excess below $2 \mathrm{keV}$, a complex iron K-line features, and a cutoff around $100 \mathrm{keV}$. The hard cutoff can be interpreted by a thermal comptonization model as well as a reflection component. The resent wide band and high quality X-ray data suggest that the existence of two thermal comptonization components by hot coronae with a low and high optical depth, respectively (Takahashi et al. 2008; Makishima et al. 2008).

We examined the diffrerence of this two comptonization components between dip and non-dip spectra.

\section{Observation \& data reduction}

We analyzed RXTE data, observed from 2000-01-05 00:50:56 to 2000-01-10 22:02:08 (1 orbital cycle), which is the same data that Feng \& Cui (2002) reported the characteristics of the observed dips. In this observation, Cyg X-1 was in the low/hard state, and the net exposure was $95.5 \mathrm{ksec}$. The instrument of the RXTE consists of PCA (3-25 keV) and HEXTE (20-150 keV). We used standard2a in the archive data for both PCA and HEXTE. PCA consists of fi ve PCUs, we used three of that (PCU0, 2 ,3), and selected all layers for each PCUs. Then, we extracted light curves and spectra in the standard procedure.

\section{Light curve \& hardness ratio}

We extracted light curves for three energy bands (2-5 keV, 5-10 keV, 10-30 keV), and calculated hardness ratios between them (Fig. 1) : HR1 (Hardness Ratio of 5-10 keV count rate to 2-5 $\mathrm{keV}$ count rate) and HR2 (5-10 keV to $10-30 \mathrm{keV})$. HR1 sometimes shows abrupt increases, and we defi ned the increases as intensity dip (Fig. 2), which are corresponding to the "type A dips" (Feng \& Cui 2002). The net exposure time of dips is $1.6 \mathrm{ksec}$.

\section{Spectral analysis}

Figure 3 shows a ratio of dip spectrum to non-dip one. The dip spectrum below $\sim 5 \mathrm{keV}$ is absorbed strongly. In 20-150 keV energy range, it even reduced $\sim 2 \%$ from non-dip spectrum. This intensity reduction in the high energy region can be interpreted that the electron scatterings by the absorbing matter scatter out the X-rays from the line of sight.

We fi tted both dip and non-dip spectrum with two CompPS model (Poutanen \& Svensson 1996), and added a gaussian function for the Fe K emission line. We fi xed the temperature of seed photon $k T_{\mathrm{bb}}=0.19$, which is the same value as Suzaku result (Makishima et al. 2008), because it can not be determined by our data. We assumed the innner and outer radius for reflection, 


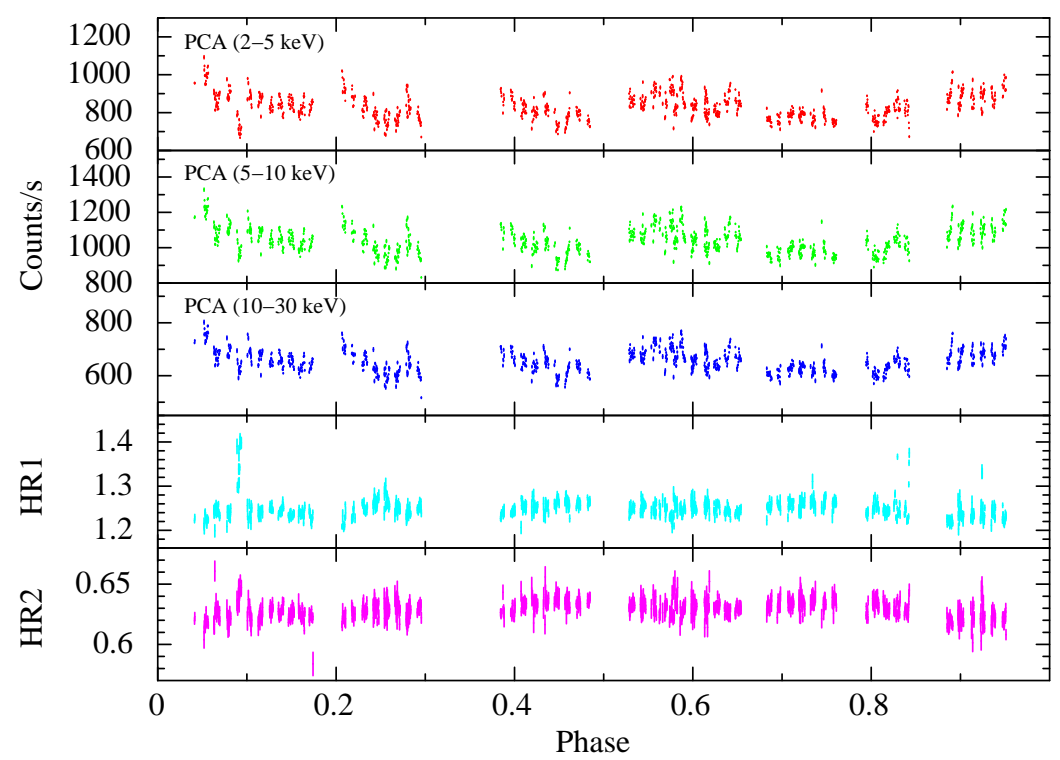

Figure 1: Lightcurve and hardness ratio for all observation. HR1 $=5-10 \mathrm{keV}$ count rate/2-5 keV count rate, $\mathrm{HR} 2=10-30 \mathrm{keV}$ count rate $/ 5-10 \mathrm{keV}$ count rate.
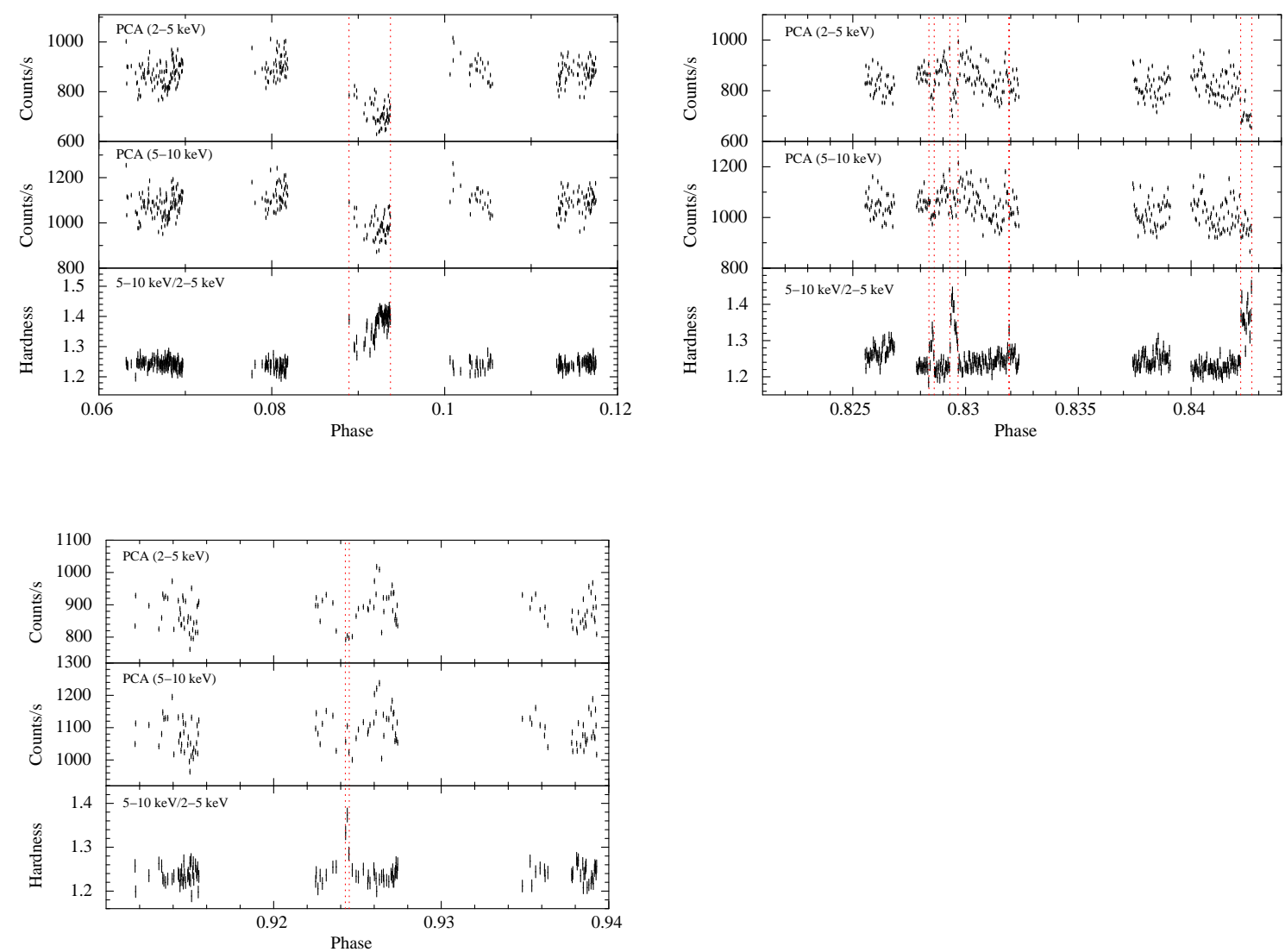

Figure 2: Enlargement of Fig. 1. The observations between red dotted lines are when intensity dips occured. 
$R_{\text {in }}^{\text {ref }}=2500 R_{g}, R_{\text {out }}^{\text {ref }}=10^{6} R_{g}$, respectively, the temperature of disk $T_{\text {disk }}=10^{6} \mathrm{~K}$ and inclination angle $=45^{\circ}$ (Abubekerov et al. 2004). We set the parameters for comptonization and reflection free for non-dip spectrum, while for dip spectrum, they are fi xed as values given by non-dip fi tting.

The spectra with the best fit model curves and their residuals are shown in figure. 4. The spectra can be reasonably fi tted with the $\xi^{2} /$ d.o.f values of 55/76 and $68 / 80$, respectively. The best fi t parameters are summarized in table 1 .

\section{Discussion}

We fi tted the non-dip spectrum with the same model used by the Suzaku data (Makishima et al. 2008) and the Beppo SAX data (Frontera et al. 2001), the double CompPS model. The resultant parameters are roughly middle of these two observations and the double CompPS model can well describe the Low/Hard state spectrum of Cygnus X-1.

The dip spectrum was also fi tted by the same model, but we made the absorption column of the both soft and hard components to be free. Then the extra $H_{\mathrm{N}}$ value of the soft component was well determined to be $\sim 10^{22} \mathrm{H}$ atoms $\mathrm{cm}^{-2}$, but that of the hard component was not well constrained from the spectral fi tting. However, we found that the intensity of the hard component decreased with $\sim 2 \%$ during the dip (see Fig. 2). If the absorbing matter, which is responsible to the electron scatting, has a enough small solid angle from the X-ray emission region. The electron column density can be estimated to be $3.0 \times 10^{22} \mathrm{~cm}^{-2}$. This value correspond to be a $\sim 2.3 \times 10^{22} \mathrm{H}$ atoms $\mathrm{cm}^{-2}$ for the solar abundance.

This result of the large scatting matter of the hard component can be interpreted by some reasons. First, the absorbing matter may be partially ionized. However, Kitamoto et al. (1984) reported the iron K-edge energy of less than $7.18 \pm 0.18 \mathrm{keV}$, suggesting insignifi cant difference of the absorption by the light elements from their neutral case. Secondly, the higher component is concentrated into a center of the $\mathrm{X}$-ray emission region and effectively has a high chance probability of the deep dips. If this is a case, much statistically high observation and much deep dips might provide us a unprecedented information of the X-ray emission region of the black hole accretion disk.

\section{References}

[1] Abubekerov et al. 2004, Astron. Rep, 48, 550

[2] Brocksopp et al. 1999, A\&A, 343, 861

[3] Feng \& Cui, 2002, ApJ, 564, 953

[4] Frontera et al. 2001, ApJ, 546, 1027

[5] Kitamoto et al. 1984, PASJ, 36, 731

[6] Makishima et al. 2008, PASJ, 60, 585

[7] Poutanen \& Svensson, 1996, ApJ, 470, 249

[8] Takahashi et al. 2008, PASJ, 60, 69 


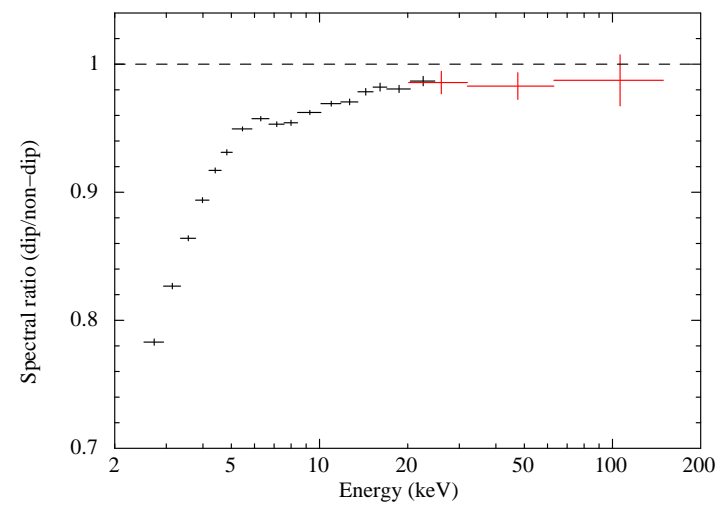

Figure 3: Spectral ratio of dip spectrum to non-dip one. White and red crosses show PCA and HEXTE data, respectively.
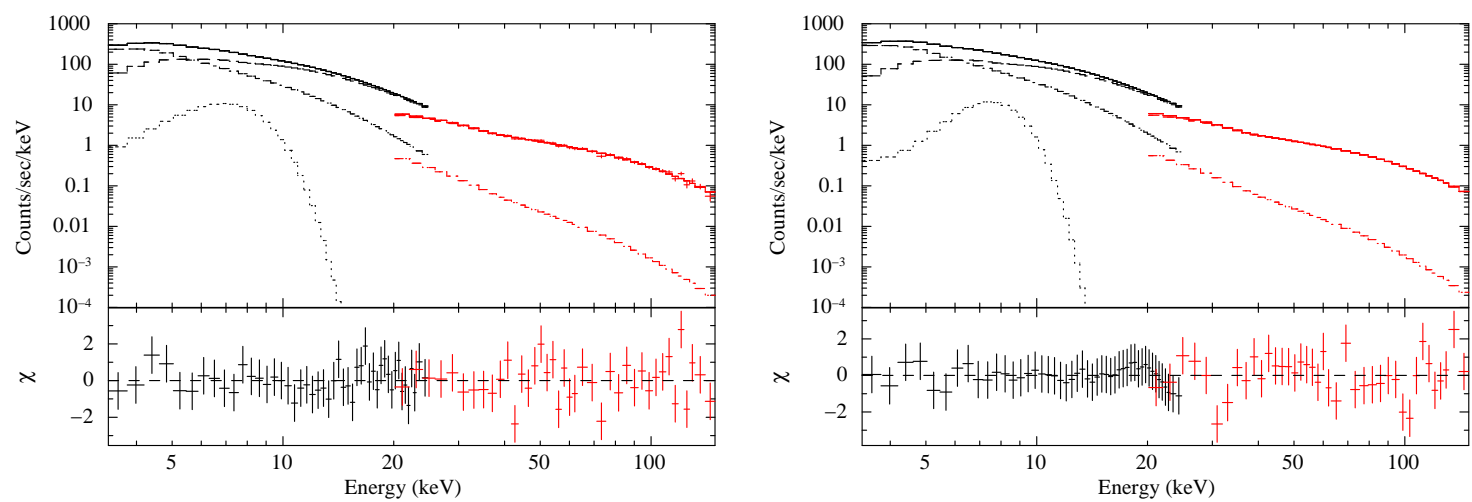

Figure 4: Dip spectrum (left) and non-dip one (right) fitting with (CompPS * wabs + ( CompPS + gaussian) * wabs ) model

Table 1: Best fit parameters for (CompPS + gaussian $) *$ wabs + (CompPS + gaussian $) *$ wabs $)$ model

\begin{tabular}{|c|c|c|c|}
\hline Component & parameter & non-dip & $\operatorname{dip}$ \\
\hline \multirow[t]{4}{*}{ compPS (hard) } & $k T_{\mathrm{bb}}(\mathrm{keV})$ & 0.19 (fi xed) & 0.19 (fi xed) \\
\hline & $T_{\mathrm{e}}(\mathrm{keV})$ & $72.3 \pm 2.0$ & 72.3(fi xed) \\
\hline & optical depth & $1.75 \pm 0.05$ & 1.75 (fi xed) \\
\hline & $R_{\text {in }}^{\text {seed }}(\mathrm{km})$ & $306 \pm 41$ & $304 \pm 17$ \\
\hline wabs (hard) & $N_{\mathrm{H}}\left(10^{22} \mathrm{~cm}^{-2}\right)$ & $14.8 \pm 1.1$ & $13.8 \pm 0.8$ \\
\hline \multirow[t]{3}{*}{ compPS (soft) } & $k T_{\mathrm{e}}(\mathrm{keV})$ & $72.3 \pm 2.0$ & 72.3 (fi xed) \\
\hline & optical depth & $0.18 \pm 0.01$ & 0.18 (fi xed) \\
\hline & $R_{\text {in }}^{\text {seed }}(\mathrm{km})$ & $1882 \pm 41$ & $1738 \pm 36$ \\
\hline wabs (soft) & $N_{\mathrm{H}}\left(10^{22} \mathrm{~cm}^{-2}\right)$ & $2.0 \pm 0.2$ & $2.6 \pm 0.2$ \\
\hline \multirow[t]{3}{*}{ reflection } & $\Omega / 2 \pi$ & $0.24 \pm 0.01$ & 0.24 (fi xed) \\
\hline & $\xi\left(\mathrm{erg} \mathrm{cm} \mathrm{s}^{-1}\right)$ & $0($ fi xed) & $0($ fi xed $)$ \\
\hline & $R_{\text {in }}^{\text {ref }}\left(R_{\mathrm{g}}\right)$ & 2500(fi xed) & 2500(fi xed) \\
\hline \multirow[t]{3}{*}{ gaussian } & centroid $(\mathrm{keV})$ & $7.07 \pm 0.10$ & $6.38 \pm 0.26$ \\
\hline & sigma (keV) & $1.05 \pm 0.13$ & $1.41 \pm 0.26$ \\
\hline & eq. width (eV) & 612 & 869 \\
\hline fi t goodness & $\chi_{v}^{2}(v)$ & $0.73(76)$ & $0.85(80)$ \\
\hline
\end{tabular}

